Draft Version November 8, 2018

Preprint typeset using $\mathrm{LAT}_{\mathrm{E} X} \mathrm{X}$ style emulateapj v. 11/12/01

\title{
THE 2008 LUMINOUS OPTICAL TRANSIENT IN THE NEARBY GALAXY NGC $300^{1}$
}

\author{
Howard E. Bond ${ }^{2}$ Luigi R. Bedin, ${ }^{2}$ Alceste Z. Bonanos, ${ }^{2,3}$ Roberta M. Humphreys, ${ }^{4}$ \\ L.A.G. Berto Monard, ${ }^{5}$ José L. Prieto, ${ }^{6}$ and Frederick M. Walter ${ }^{7}$ \\ Draft version November 8, 2018
}

\begin{abstract}
A luminous optical transient (OT) that appeared in NGC 300 in early 2008 had a maximum brightness, $M_{V} \simeq-12$ to -13 , intermediate between classical novae and supernovae. We present ground-based photometric and spectroscopic monitoring and adaptive-optics imaging of the OT, as well as pre- and post-outburst space-based imaging with HST and Spitzer. The optical spectrum at maximum showed an F-type supergiant photosphere with superposed emission lines of hydrogen, Ca II, and [Ca II], similar to the spectra of low-luminosity Type IIn "supernova impostors" like SN 2008S, as well as cool hypergiants like IRC +10420 . The emission lines have a complex, double structure, indicating a bipolar outflow with velocities of $\sim 75 \mathrm{~km} \mathrm{~s}^{-1}$. The luminous energy released in the eruption was $\sim 10^{47}$ ergs, most of it emitted in the first 2 months. By registering new HST images with deep archival frames, we have precisely located the OT site, and find no detectable optical progenitor brighter than broad-band $V$ magnitude 28.5. However, archival Spitzer images reveal a bright, non-variable mid-IR pre-outburst source. We conclude that the NGC 300 OT was a heavily dust-enshrouded luminous star, of $\sim 10-$ $15 M_{\odot}$, which experienced an eruption that cleared the surrounding dust and initiated a bipolar wind. The progenitor was likely an $\mathrm{OH} / \mathrm{IR}$ source which had begun to evolve on a blue loop toward higher temperatures, but the precise cause of the outburst remains uncertain.
\end{abstract}

Subject headings: stars: variables: other — galaxies: individual (NGC 300) — galaxies: stellar content — stars: individual (NGC 300 OT) — stars: winds, outflows - supernovae: general

\section{INTRODUCTION: TRANSIENTS IN THE NOVA-SUPERNOVA GAP}

In recent years, eruptive objects with maximum luminosities intermediate between those of classical novae $(\mathrm{CNe})$ and supernovae $(\mathrm{SNe})$ have been discovered in increasing numbers, as surveys for Galactic and extragalactic transients are made with greater depth and sky coverage by professional and amateur astronomers. The term "supernova impostors" was introduced by Van Dyk et al. (2000) following the outburst of SN 1997bs. SN 1997bs was classified as a SN IIn event, "n" denoting narrow emission lines during outburst, in contrast to the high ejection velocities of typical SNe. However, it reached an absolute magnitude at maximum of only $M_{V} \simeq-13.8$, more than 3 mag fainter than a typical core-collapse SN. Van Dyk et al. argued that SN 1997bs was instead a "superoutburst" of a massive luminous blue variable (LBV), analogous to those experienced by the Galactic objects P Cygni and $\eta$ Carinae (see Humphreys, Davidson, \& Smith 1999). The subject of intrinsically faint SNe IIn and $\eta$ Car analogs has been summarized by Van Dyk (2005) and Smith et al. (2008). More recently, SN 2008S, which had a SN IIntype spectrum but reached only $M_{R} \simeq-13.9$, has been discussed by Thompson et al. (2008) and Smith et al. (2008). Prieto et al. (2008) have identified the precursor of SN 2008S in pre-outburst mid-IR images obtained with the Spitzer Space Telescope, demonstrating that the progenitor was a luminous star deeply embedded in circumstellar dust.

V838 Monocerotis is the prototype of an apparently separate class of transients also lying in the CN-SN gap. V838 Mon erupted in 2002, illuminating a spectacular light echo that provided a geometric distance (Sparks et al. 2008) implying an absolute magnitude at maximum of $M_{V}=-9.8$. Unlike a CN, V838 Mon became progressively redder during its outburst, eventually becoming enshrouded in circumstellar dust. Its outburst light curve (Bond et al. 2003) was very different from that of a SN or LBV eruption, showing a series of four maxima separated by about a month each. The cause of this outburst remains uncertain, but a leading suggestion is that it was a stellar collision or merger (e.g., Tylenda \& Soker 2006, Corradi \& Munari 2007, and references therein).

Possible extragalactic analogs of V838 Mon-although this interpretation is controversial - include the "M31 red variable" ("M31 RV") which reached $M_{V, \max } \simeq-9.4$

${ }^{1}$ Based in part on observations with the NASA/ESA Hubble Space Telescope obtained at the Space Telescope Science Institute, and from the data archive at STScI, which is operated by the Association of Universities for Research in Astronomy, Inc., under NASA contract NAS5-26555; in part on archival data obtained with the Spitzer Space Telescope, which is operated by the Jet Propulsion Laboratory, California Institute of Technology, under a contract with NASA; in part on observations obtained with the 6.5-m Magellan Clay Telescope located at Las Campanas Observatory, Chile; and in part on observations obtained with the SMARTS Consortium 1.3- and 1.5-m telescopes located at Cerro Tololo Interamerican Observatory, Chile.

2 Space Telescope Science Institute, 3700 San Martin Dr., Baltimore, MD 21218; [bond, bedin, abonanos]@stsci.edu

${ }^{3}$ Giacconi Fellow

${ }^{4}$ Astronomy Department, University of Minnesota, Minneapolis, MN 55455; roberta@aps.umn.edu

${ }^{5}$ Bronberg Observatory, PO Box 11426, Tiegerpoort 0056, South Africa

${ }^{6}$ Department of Astronomy, The Ohio State University, 140 W. 18th Ave., Columbus, OH 43210; prieto@astronomy.ohio-state.edu

7 Department of Physics \& Astronomy, Stony Brook University, Stony Brook, NY 11704; fwalter@astro.sunysb.edu 
(Bond \& Siegel 2006 and references therein), and a luminous red transient in M85 (Rau et al. 2007; Kulkarni et al. 2007), which attained $M_{V \text {, max }} \simeq-13$. M31 RV and the M85 transient appear to have occurred in old populations, suggesting that they did not arise from very massive progenitors.

In this Letter, we report observations of a luminous transient discovered in 2008 May in the nearby spiral galaxy NGC 300. We obtained extensive ground-based imaging, photometry, and spectroscopy, and we also investigated pre- and post-outburst space-based optical and infrared images of the transient. This Letter presents an overview of our results, along with a preliminary discussion of the nature of this remarkable object. Subsequent publications will give fuller details. We designate the object as the "NGC 300 optical transient," hereafter "NGC 300 OT."

\section{DISCOVERY AND OUTBURST LIGHT CURVE}

The outburst of NGC 300 OT was discovered by Monard (2008) during his SN search program with the Bronberg Observatory $0.3-\mathrm{m}$ telescope equipped with a CCD camera. The OT was detected at broad-band magnitude 14.3 on a frame obtained on 2008 May 14 (all dates in this paper are UT). Subsequent inspection of an image from 2008 April 24, taken in morning twilight shortly after NGC 300 had emerged from behind the Sun, showed that the OT was already rising, at $\sim 16.3 \mathrm{mag}$. It was fainter than 18 mag on 2008 February 8, and on all previous Bronberg observations.

NGC 300 is an SAd spiral in the Sculptor Group, located just outside the Local Group. The OT lies in a spiral arm with active star formation. We adopt a distance modulus of $(m-M)_{0}=26.37(d=1.88 \mathrm{Mpc})$, based on Cepheids (Gieren et al. 2005) and the red-giant tip (Rizzi et al. 2006).

At maximum the OT was much fainter than a typical $\mathrm{SN}$, but brighter than any CN. Because of our interest in luminous red transients, we began a program of photometric and spectroscopic monitoring, using the 1.3- and $1.5-\mathrm{m}$ telescopes at Cerro Tololo Interamerican Observatory operated by the SMARTS Consortium 8 The photometric monitoring in particular was intensive at first, because of our expectation that the OT might exhibit periodic spikes, like those during the outburst of V838 Mon (Bond et al. 2003).

We used the ANDICAM optical/near-IR direct camera (DePoy et al. 2003) on the SMARTS 1.3-m with CCD and IR detectors, operated simultaneously, for the $B V R I$ and $J H K$ photometry. The BVRI magnitudes of the OT were determined differentially with respect to a nearby comparison star, calibrated on photometric nights using Landolt (1992) standard fields. The JHK magnitudes are differential with respect to the same star, calibrated using its 2MASS photometry. The J2000 position of the OT, based on astrometry of ANDICAM frames calibrated against the USNO-NOMAD catalog, is RA $=00: 54: 34.51$, Dec $=-37: 38: 31.4$, with errors in each coordinate of about \pm 0.2 .

Figure 1 shows the BVRIJHK light curve. Unfortunately, the rise to maximum was poorly covered, but it appears that it was more rapid than the subsequent slower decline. The brightest $V$ magnitude we measured was 14.69, on the first night of SMARTS observations (2008 May 15), corresponding to $M_{V, 0}=-12.0$ to -12.9 for the adopted distance and a reddening lying in the range $E(B-V)=0.1$ to 0.4 (see $\S 3.1$ ). Following maximum light, the OT has declined smoothly in brightness at all wavelengths, while becoming steadily redder. For example, $V-K$ evolved from 3.1 in mid-May to 7.4 at the beginning of 2008 December. The rate of decline in the optical lessened around the beginning of 2008 September, but at this writing the brightness continues to decrease in both the optical and near-IR. The $R$ magnitudes are not fading as rapidly as in the other filters, due to strong $\mathrm{H} \alpha$ emission.

\section{SPECTROSCOPY}

\subsection{Low Resolution}

We obtained low- and moderate-resolution optical spectroscopy of NGC 300 OT throughout its outburst, using the SMARTS 1.5-m Cassegrain spectrograph. Our first low-resolution spectrum was observed on 2008 May 15 (Bond, Walter, \& Velásquez 2008) and is shown in Figure 2 (top spectrum; resolution $17.2 \AA$ ). This spectrum exhibits strong emission at $\mathrm{H} \alpha, \mathrm{H} \beta$, the $\mathrm{Ca}$ II triplet at $8542-$ 8498-8662 $\AA$, and the unusual forbidden [Ca II] doublet at 7291-7323 A. The Balmer lines are only slightly resolved at the velocity resolution $\left(790 \mathrm{~km} \mathrm{~s}^{-1}\right)$ of the spectrum. The underlying continuum resembles a high-luminosity Ftype supergiant, with $\mathrm{Ca}$ II $\mathrm{H}$ and $\mathrm{K}$ seen in absorption along with several weaker luminosity-sensitive lines such as the O I triplet at $7774 \AA$. Our photometry at maximum and shortly afterwards yields $B-V \simeq 0.8$, implying that the inter- and circumstellar extinction could be as high as $E(B-V) \simeq 0.4$, since a mid- to late F-type supergiant normally has $(B-V)_{0} \simeq 0.4$. The mean foreground reddening of NGC 300 is $E(B-V) \simeq 0.1$ (Gieren et al. 2005).

This spectrum is clearly not that of a $\mathrm{CN}$ nor a typical SN. With strong Ca II and [Ca II] emission and an F-type absorption spectrum, it resembles that of the Type IIn supernova impostor SN 2008S (Steele et al. 2008; Smith et al. 2008). It is also closely similar to the spectra of the well-studied post-red supergiant IRC +10420 (Jones et al. 1993; Humphreys, Davidson, \& Smith 2002), Variable A in M33 in its recent quiescent state (Humphreys et al. 2006), and V838 Mon near its maximum (e.g., Wisniewski et al. 2003). These F-type supergiant spectra with superposed narrow emission lines of relatively low excitation are indicative of eruptions that produce an optically thick wind or slowly expanding envelope.

As the OT declined, its spectrum became dominated by the $\mathrm{H} \alpha$ and $\mathrm{Ca}$ II emission. One example, selected from our monitoring program, is the bottom spectrum shown in Figure 2, obtained on 2008 August 23. By this date, the continuum had faded dramatically and reddened somewhat, but the strong emission remained.

\subsection{Moderate Resolution}

We also obtained moderate-resolution (3.1 $\AA$ ) spectra regularly with the SMARTS $1.5-\mathrm{m}$ spectrograph, and on three occasions with the Magellan 6.5-m telescope and

8 SMARTS is the Small and Medium Aperture Research Telescope System; see http://www.astro.yale.edu/smarts/ 
MagE echellette spectrograph (Marshall et al. 2007; resolution $1.5 \AA$ at Ca II, $1.1 \AA$ at $\mathrm{H} \alpha$ ): 2008 July 6, Aug 30, and Sep 1. These spectra show that the Ca II triplet, $\mathrm{H} \alpha$, and $\mathrm{H} \beta$ have double-peaked emission lines, with the blue component being the stronger.

Figure 3 shows the $\mathrm{H} \alpha$ and Ca II emission features from the Magellan echellette spectra on 2008 July 6 (top spectrum) and the average of the August 30 and September 1 observations (bottom spectrum), and illustrates the double structure of these lines. Double emission is usually attributed either to a bipolar outflow or a rotating disk, but in the case of an eruption, the double lines are most likely formed in a bipolar wind. IRC +10420 shows very similar double-peaked emission and has strong independent evidence for a bipolar outflow (Davies et al. 2007; Patel et al. 2008).

The peak of the OT's primary blue component slowly increases in strength with time relative to the red component, supporting our conclusion that the outflow is bipolar, with the nearer, blue-shifted lobe expanding towards us. The Doppler velocities measured from both the hydrogen and Ca II emission lines in the echellette spectra indicate expansion velocities for the primary wind of $\sim 70-80 \mathrm{~km} \mathrm{~s}^{-1}$ with respect to the star's systemic velocity of about $+196 \mathrm{~km} \mathrm{~s}^{-1}$. There is evidence as well for additional emission components, appearing as shoulders or secondary bumps on the primary blue and red peaks at velocities of $\approx 160 \mathrm{~km} \mathrm{~s}^{-1}$.

A wind velocity of $\sim 75 \mathrm{~km} \mathrm{~s}^{-1}$ is typical of the winds of F-type supergiants, and is somewhat lower than the winds associated with LBVs in eruption. It is very similar to the expansion velocity of $\sim 60 \mathrm{~km} \mathrm{~s}^{-1}$ of IRC +10420 (Jones et al. 1993; Humphreys et al. 2002). By contrast, the [Ca II] lines, formed in a very low-density region, do not show a double-peaked structure; they probably arise in moredistant and slower-moving material, likely formed before the current eruption.

\section{HST AND VLT PRE- AND POST-OUTBURST IMAGING}

We imaged the site of the OT twice with the Hubble Space Telescope $(H S T)$, in the Director's Discretionary (DD) program GO-11553 (PI: Bond). We used the Wide Field Planetary Camera 2 (WFPC2) with the F450W and F814W filters; observations were made on 2008 June 9 and September 1. In addition, the HST archive contains two sets of deep observations made before the outburst, obtained with the Wide Field Channel of the Advanced Camera for Surveys (ACS) and the F435W, F475W, F555W, F606W, and F814W filters. The ACS observations were made on 2002 December 25 (GO-9492, PI: F. Bresolin) and 2006 November 8-10 (GO-10915, PI: J. Dalcanton). We used these data to locate the OT precisely, search for a progenitor object, study the surrounding stellar population, and set limits on a light echo from the outburst.

In addition to the $H S T$ data, we were awarded DD time on the ESO Very Large Telescope (VLT) to obtain near-IR imaging of the OT, using the adaptive-optics $\mathrm{NaCo}$ camera (Lenzen et al. 2003; Rousset et al. 2003). The VLT/ $\mathrm{NaCo}$ images were obtained on 2008 June 6 .

\subsection{Astrometry and Pre-Outburst Magnitude Limits}

The WFPC2 exposure times for our DD observations ranged from 5 to $260 \mathrm{~s}$, chosen so as not to saturate the image of the OT, but to show enough of the surrounding star field for precise registration with the pre-outburst ACS frames. The archival ACS frames are considerably deeper, with total exposure times of 1080, 2976, 1080, 3030, and $4524 \mathrm{~s}$ in F435W, F475W, F555W, F606W, and F814W, respectively. The VLT/NaCo J-band frames were four $600 \mathrm{~s}$ exposures, combined in the ESO pipeline to produce a single image.

Figure 4 shows a montage of post- and pre-outburst images. The OT lies amid a rich field of resolved stars. Remarkably, however, there is no significant detection of a progenitor object at the OT's precise location. The two nearest reliably detected sources lie about 0 "' 15 and 0 '.25 from the site, well in excess of our registration error of about $0 . \prime 01$. These two stars are red giants with F606W magnitudes of about 26.3 and 27.0. In the deepest stacked pre-outburst images there are suggestions of local maxima at the OT site, but these are all consistent with noise. From the three deepest ACS stacked images, we find the following limiting magnitudes (Vega-mag scale, $3 \sigma$ upper limits) for the OT progenitor: 28.3 (F475W), 28.5 (F606W), and 26.6 (F814W). Similar limiting magnitudes were reported earlier, based on registering the 2006 ACS images with ground-based Magellan/Clay 6.5-m images, by Berger \& Soderberg (2008), but with larger positional uncertainties.

The OT was thus very unlike a typical core-collapse SN, for which luminous progenitor stars are being found relatively easily in archival $H S T$ images at distances greater than that of NGC 300 (e.g., Smartt et al. 2008 and references therein), nor was it similar to erupting LBVs, which even in quiescence have high optical luminosities (e.g., Humphreys \& Davidson 1994).

\subsection{The Surrounding Young Stellar Population}

The HST ACS images also provide deep CMDs for the environment surrounding the OT. These will be presented in detail in a subsequent publication, but we note that there is a rich population of young objects in the immediate vicinity of the OT. For example, within $2.5(\sim 23 \mathrm{pc})$ of the OT site there are over a dozen blue main-sequence stars brighter than about $\mathrm{F} 606 \mathrm{~W}$ magnitude 26, reaching as bright as $M_{V} \approx-3$ (corresponding to B1 stars of $\left.\approx 14 M_{\odot}\right)$. The surrounding spiral-arm field is rich in blue clusters and associations.

\subsection{Search for Light Echo}

We compared the post-outburst HST images of 2008 June 9 and September 1 in search of an expanding light echo, similar to the one surrounding V838 Mon (e.g., Bond 2007, Sparks et al. 2008, and references therein). Although a light echo of the surface brightness and angular size of V838 Mon's, moved to the distance of NGC 300, would have been detectable at $H S T$ resolution, no echo is seen.

\section{Spitzer PRE-OUTBURST OBSERVATIONS: AN ENSHROUDED RED SUPERGIANT}

The very faint limits on the pre-outburst optical brightness of NGC 300 OT initially suggested to us that there was a similarity with V838 Mon, whose progenitor was 
Bond et al.

also optically inconspicuous (e.g., Afşar \& Bond 2007, and references therein). However, the very different light curve and spectroscopic behavior of the OT as its outburst proceeded casts doubt on this parallel.

Moreover, a striking difference emerged when one of us (Prieto 2008) reported that the progenitor of the OT was detected as a bright mid-IR source in archival observations obtained with Spitzer on 2003 November 21 (PI: G. Helou) and 2007 December 28 (PI: R. Kennicutt). The source was present in all four IRAC bands $(3.6,4.5,5.8$, and $8 \mu \mathrm{m})$, and in the MIPS $24 \mu \mathrm{m}$ band. A black-body fit to the 3.6$24 \mu \mathrm{m}$ fluxes gives the following results for the luminosity, effective temperature, and radius of the OT progenitor: $L_{\mathrm{bb}}=5.5 \times 10^{4} L_{\odot}, T_{\mathrm{bb}}=350 \mathrm{~K}$, and $R_{\mathrm{bb}}=300 \mathrm{AU}$. These are consistent with a heavily dust-enshrouded red supergiant of $\sim 10-15 M_{\odot}$ (e.g., Maeder \& Meynet 2000). There was no variability of the progenitor between 2003 and 2007. Further detailed discussion of the Spitzer observations is given by Thompson et al. (2008).

\section{NATURE OF THE LUMINOUS TRANSIENT IN NGC 300}

A picture thus emerges in which a luminous massive star, optically inconspicuous because it was deeply embedded in a dusty envelope, underwent a sudden outburst that cleared most of the surrounding dust. In many respects, NGC 300 OT has behaved remarkably similarly to the supernova impostor SN 2008S (see $\S 1$ ). SN 2008S was also heavily enshrouded by dust prior to its outburst, with an intrinsic luminosity of $3.5 \times 10^{4} L_{\odot}$ based on its IR SED, implying an initial mass of $\sim 10 M_{\odot}$.

The energy released in the OT's eruption was relatively modest. Integrating over the extinction-corrected visual light curve, beginning with the initial detection in 2008 April, and assuming that the bolometric correction is zero, we find that the total energy released was $\sim 0.8^{-}$ $2 \times 10^{47}$ ergs. This is similar to the energy radiated by SN 2008S, $\sim 6 \times 10^{47}$ ergs (Smith et al. 2008), but considerably less than the $\sim 10^{50}$ ergs emitted in $\eta$ Car's great eruption, and of course the $\sim 10^{51} \mathrm{ergs}$ in true core-collapse SNe.

Given the large amounts of pre-outburst obscuring dust, both NGC 300 OT and SN 2008S must have experienced significant mass loss as post-main-sequence stars, and were most likely red supergiants (RSGs), post-RSGs, or conceivably lower-mass stars at the tip of the AGB just before the outbursts. Thompson et al. (2008) have suggested that these events may represent a new class of low-luminosity outbursts arising from stars in the mass range $\sim 8-11 M_{\odot}$.
By comparison with multi-epoch Spitzer IRAC observations in M33, they have identified a group of rare, reddened sources that they call extreme-AGB stars, which may be the progenitor class in a short-lived stage lasting only $\leq 10^{4}$ yr immediately preceding the outbursts. There are also many enshrouded OH/IR stars in our own Galaxy, including AGB-tip stars and red supergiants with very red mid-IR colors; some may be as red as the progenitors of the OT and SN 2008S.

Here we suggest that NGC $300 \mathrm{OT}$, and by analogy SN 2008S, were OH/IR stars before their eruptions. Given both objects' lack of obvious variability from Spitzer observations several years apart, these OTs were not Mira variables just before outburst. They had presumably left the region of RSGs and AGB stars in the HR diagram, and were on a blue loop back to warmer temperatures. The NGC 300 OT's bipolar wind at $\approx 75 \mathrm{~km} \mathrm{~s}^{-1}$ also supports a warmer temperature for the progenitor. During this transition, both stars reached a state in which they exceeded the Eddington limit for their luminosities and masses and suddenly initiated outflows, bipolar in the case of NGC 300 OT. Exactly why this should happen remains uncertain: the eruptions may have resulted from some type of as-yet unexplained failed supernova, a binary merger, or a photospheric eruption.

Continued observations of the NGC 300 OT as it fades are especially important. We currently observe the spectrum of its wind, but if it does not form another cocoon of dust, we may eventually observe the survivor, determine its true nature, and possibly gain some insight into the cause of its eruption.

Partial support for this research was provided by NASA through grant GO-11553 from the Space Telescope Science Institute, which is operated by the Association of Universities for Research in Astronomy, Inc., under NASA contract NAS5-26555. STScI's participation in the SMARTS Consortium is supported by the STScI Director's Discretionary Research Fund. We thank M. Hernández and J. Velásquez for conducting the 1.5-m observations, J. Espinoza, A. Miranda, and J. Vasquez for the $1.3-\mathrm{m}$ observations, and Michelle Buxton and Suzanne Tourtellotte for scheduling the 1.3-m telescope. G. Pietrzyński kindly obtained one of the MagE spectra. We thank Kris Davidson, Chris Kochanek, Kris Stanek, and Todd Thompson for useful discussions.

Facilities: HST (WFPC2, ACS), Magellan:Clay, SMARTS:1.5m, SMARTS:1.3m, Spitzer, VLT:NaCo.

\section{REFERENCES}

Afsar, M., \& Bond, H. E. 2007, AJ, 133, 387

Berger, E., \& Soderberg, A. 2008, The Astronomer's Telegram, 1544, 1

Bond, H. E. 2007, in The Nature of V838 Mon and its Light Echo, ed. R. L. M. Corradi \& U. Munari (San Francisco: ASP), 130

Bond, H. E., et al. 2003, Nature, 422, 405

Bond, H. E., \& Siegel, M. H. 2006, AJ, 131, 984

Bond, H. E., Walter, F. M., \& Velásquez, J. 2008, IAU Circ., 8946, 2

Corradi, R. L. M., \& Munari, U., eds., 2007, The Nature of V838 Monocerotis and its Light Echo (San Francisco: ASP)

Davies, B., Oudmaijer, R. D., \& Sahu, K. C. 2007, ApJ, 671, 2059

DePoy, D. L., et al. 2003, Proc. SPIE, 4841, 827

Gieren, W., Pietrzyński, G., Soszyński, I., Bresolin, F., Kudritzki, R.-P., Minniti, D., \& Storm, J. 2005, ApJ, 628, 695
Humphreys, R. M., \& Davidson, K. 1994, PASP, 106, 1025

Humphreys, R. M., Davidson, K., \& Smith, N. 1999, PASP, 111, 1124

Humphreys, R. M., Davidson, K., \& Smith, N. 2002, AJ, 124, 1026

Humphreys, R. M., et al. 2006, AJ, 131, 2105

Jones, T. J., et al. 1993, ApJ, 411, 323

Kulkarni, S. R., et al. 2007, Nature, 447, 458

Landolt, A. U. 1992, AJ, 104, 340

Lenzen, R., et al. 2003, Proc. SPIE, 4841, 944

Maeder, A., \& Meynet, G. 2000, ARA\&A, 38, 143

Marshall, J. L., et al. 2008, Proc. SPIE, 7014,

Monard, L. A. G. 2008, IAU Circ., 8946, 1

Patel, M., Oudmaijer, R. D., Vink, J. S., Bjorkman, J. E., Davies, B., Groenewegen, M. A. T., Miroshnichenko, A. S., \& Mottram, J. C. 2008, MNRAS, 385, 967 
Prieto, J. L. 2008, The Astronomer's Telegram, 1550, 1

Prieto, J. L., et al. 2008, ApJ, 681, L9

Rau, A., Kulkarni, S. R., Ofek, E. O., \& Yan, L. 2007, ApJ, 659, 1536

Rizzi, L., Bresolin, F., Kudritzki, R.-P., Gieren, W., \& Pietrzyński, G. 2006, ApJ, 638, 766

Rousset, G., et al. 2003, Proc. SPIE, 4839, 140

Smartt, S. J., Eldridge, J. J., Crockett, R. M., \& Maund, J. R. 2008, arXiv:0809.0403

Smith, N., Ganeshalingam, M., Li, W., Chornock, R., Steele, T. N., Silverman, J. M., Filippenko, A. V., \& Mobberley, M. P. 2008, arXiv:0811.3929

Sparks, W. B., et al. 2008, AJ, 135, 605
Steele, T. N., Silverman, J. M., Ganeshalingam, M., Lee, N., Li, W., \& Filippenko, A. V. 2008, Central Bureau Electronic Telegrams, 1275,1

Thompson, T. A., Prieto, J. L., Stanek, K. Z., Kistler, M. D., Beacom, J. F., \& Kochanek, C. S. 2008, arXiv:0809.0510

Tylenda, R., \& Soker, N. 2006, A\&A, 451, 223

Van Dyk, S. D. 2005, The Fate of the Most Massive Stars, 332, 47

Van Dyk, S. D., Peng, C. Y., King, J. Y., Filippenko, A. V., Treffers, R. R., Li, W., \& Richmond, M. W. 2000, PASP, 112, 1532

Wisniewski, J. P., Morrison, N. D., Bjorkman, K. S., Miroshnichenko, A. S., Gault, A. C., Hoffman, J. L., Meade, M. R., \& Nett, J. M. 2003, ApJ, 588, 486

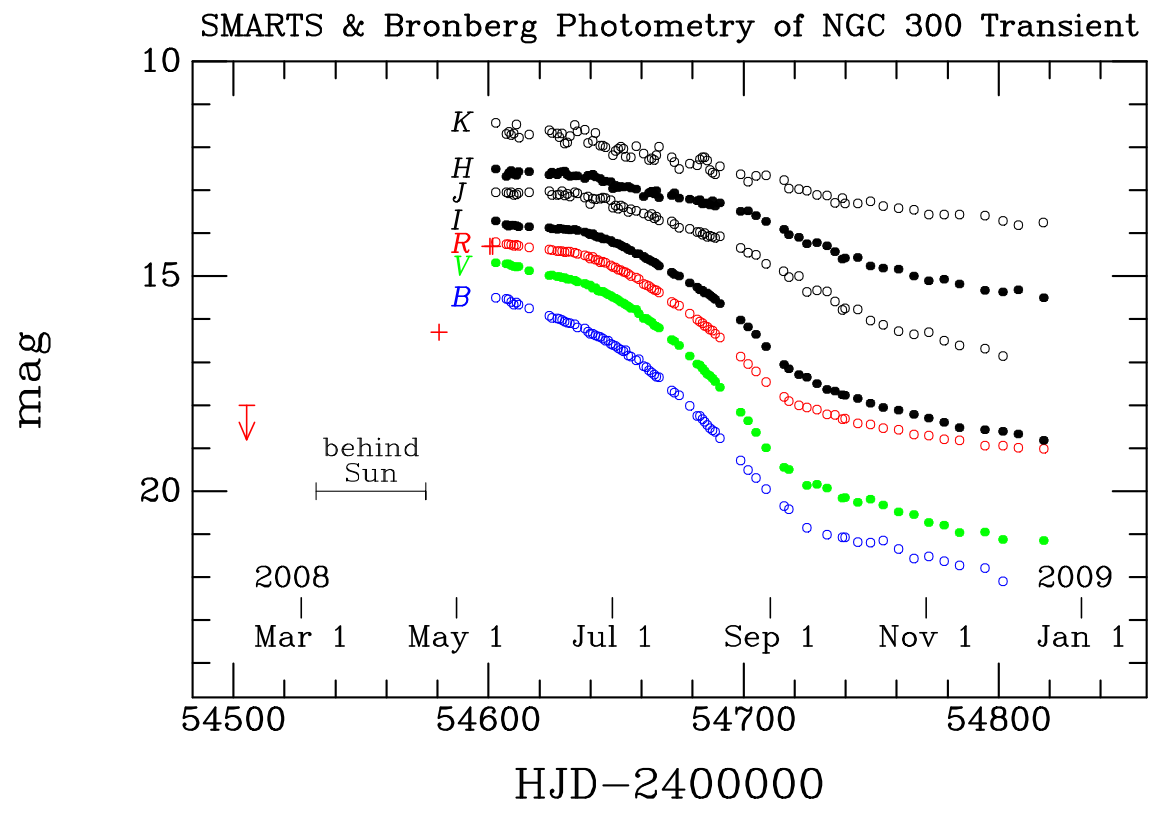

FIG. 1. - BVRIJHK light curve of the NGC 300 optical transient. SMARTS 1.3-m data are shown as open and filled circles; Bronberg

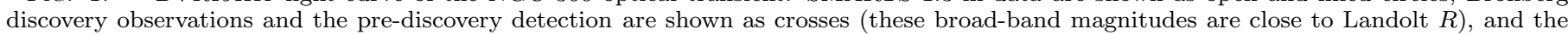
downward arrow on the left shows the upper limit in 2008 February. 


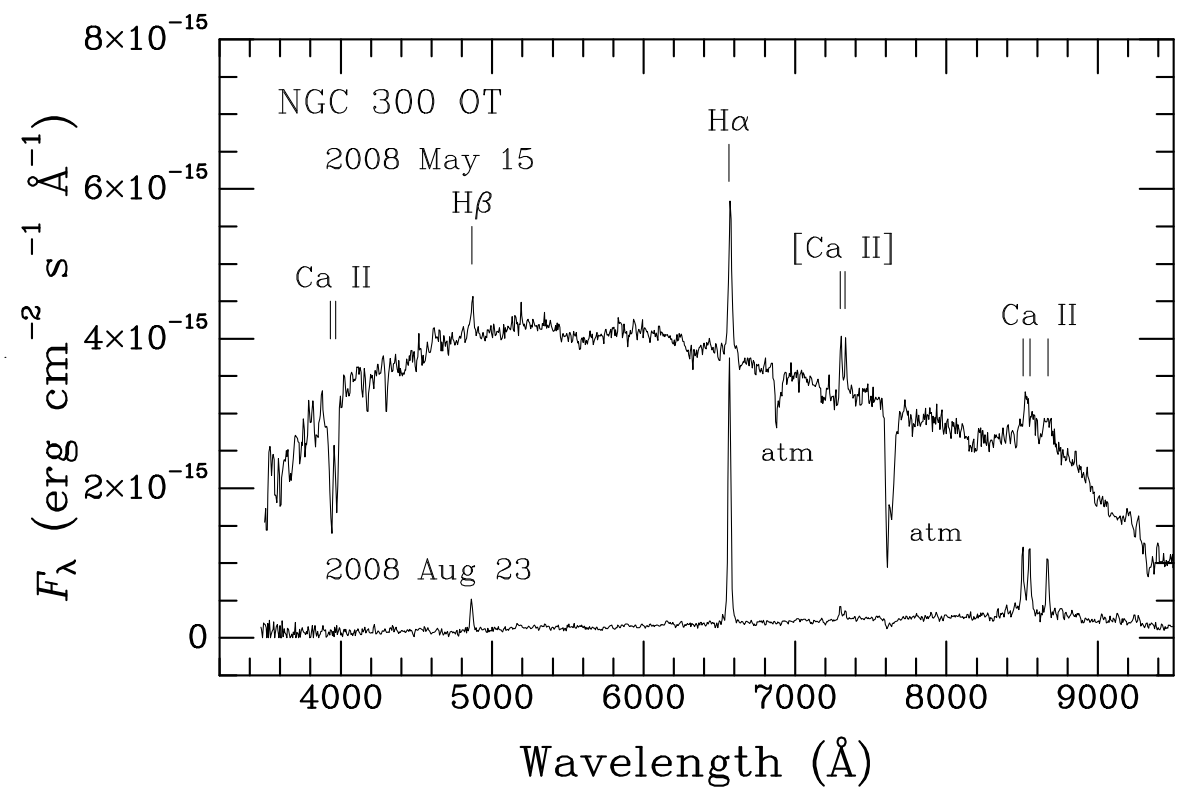

FIG. 2.- Low-resolution ( 17.2 $\AA$ ) SMARTS 1.5-m spectra of the OT on 2008 May 15 and August 23 . In the May 15 observation, note strong emission lines of $\mathrm{H}$, Ca II, and [Ca II], superposed on an F-type absorption spectrum. By August, the continuum had faded dramatically and the spectrum is dominated by the emission lines.

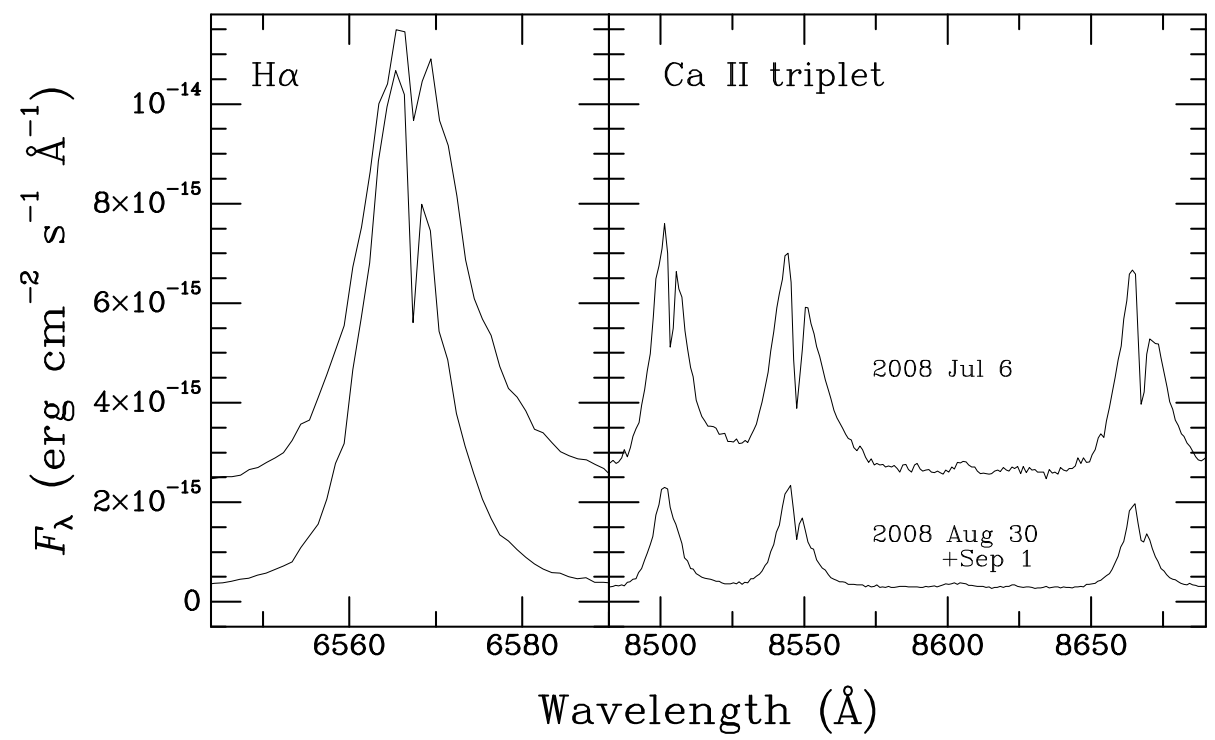

FIG. 3.- Portions of Magellan 6.5-m MagE echellette spectra (resolution $~ 1.3 \AA$ ) of the OT on 2008 July 6 and the average of two spectra taken on August 30 and September 1. The emission lines of $\mathrm{H} \alpha$ and the Ca II triplet are double, indicating a bipolar outflow. 

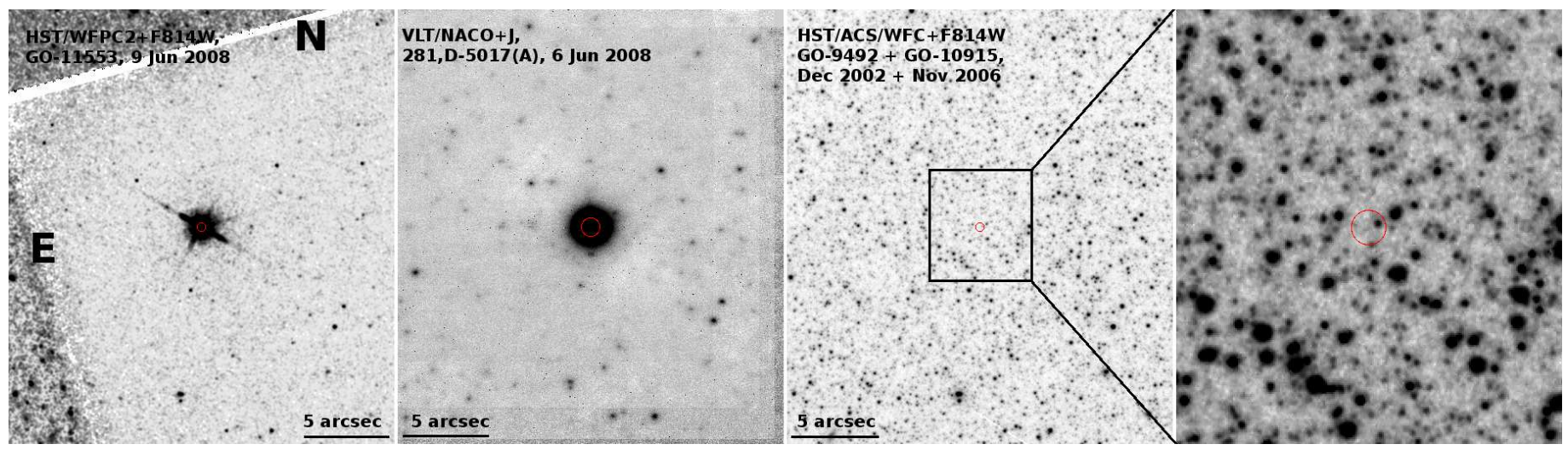

FIG. 4. - Four images showing the site of the NGC 300 OT. The first two show the OT in outburst in 2008 June and were taken with $H S T / \mathrm{WFPC} 2$ and the VLT/NaCo AO camera, using $I(\mathrm{~F} 814 \mathrm{~W})$ and $J$ filters, respectively. The third frame is a stacked HST F814W image created from pre-outburst ACS/WFC images taken in 2002 and 2006. Small red circles show the location of the OT. The fourth frame is a magnified view of the pre-outburst image, showing the location of the transient, which we have determined to $\pm 00^{\prime \prime} 01$. There is no source detected at the OT site to a limiting F814W magnitude of 26.6. North is at the top and east is on the left. Scale bars showing $5^{\prime \prime}$ are shown in the first three images, and the fourth image is $6^{\prime \prime} \times 7^{\prime \prime}$. The red circle in the fourth frame has a radius of $0{ }^{\prime \prime} 25$ (2.3 pc at the distance of NGC 300). 